\title{
INFLUÊNCIA DA ESTIMULAÇÃO SENSÓRIO-MOTORA-ORAL EM RECÉM-NASCIDOS PRÉ-TERMO
}

\section{Influence of sensory-motor-oral stimulation on preterm newborns}

\author{
Patricia Pereira Costa (1), Aneline Maria Ruedell (2), \\ Ângela Regina Maciel Weinmann ${ }^{(3)}$, Márcia Keske-Soares ${ }^{(4)}$
}

\begin{abstract}
RESUMO
Objetivo: verificar a influência da estimulação sensório-motora-oral em recém-nascidos pré-termo. Método: a amostra constou de 28 recém-nascidos pré-termo internados na Unidade de Terapia Intensiva Neonatal de um Hospital Universitário. Os sujeitos foram alocados em dois grupos, o estimulado e o controle. O grupo estimulado recebeu estimulação sensório-motora-oral duas vezes por dia. Realizaram-se duas avaliações, mensurando frequência respiratória e cardíaca, taxa de transferência, tempo de transição entre sonda e via oral plena, bem como incremento de peso. Os resultados foram analisados por meio do software STATA (10), comparando-se os grupos com o Teste T Student independente $(p<0,05)$. Resultados: não foram encontradas diferenças estatisticamente significantes entre os grupos. Conclusão: não houve influência do programa de estimulação sensório-motoraoral sobre os parâmetros avaliados.
\end{abstract}

DESCRITORES: Recém-nascido; Prematuro; Cuidado do Lactente; Comportamento de Sucção; Métodos de Alimentação

\section{INTRODUÇÃO}

Os avanços na área da atenção peri-natal têm aumentado a sobrevida de recém-nascidos prematuros, levantando questionamentos a respeito do desenvolvimento dessas crianças ${ }^{1-6}$.

A interrupção precoce da gestação pode trazer uma série de conseqüências ${ }^{7}$. Surgem dificuldades

(1) Aluna do curso de Graduação em Fonoaudiologia da Universidade Federal de Santa Maria, UFSM, Santa Maria, RS, Brasil.

(2) Fisioterapeuta; Mestranda em Distúrbios da Comunicação Humana na Universidade Federal de Santa Maria, UFSM, Santa Maria, RS, Brasil.

(3) Médica Pediatra e Neontologista; Professora do Departamento de Pediatra e Puericultura e Professora do Programa de Pós-Graduação em Distúrbios da Comunicação Humana da Universidade Federal de Santa Maria, UFSM, Santa Maria, RS, Brasil; Doutora pela Faculdade de Medicina de Ribeirão Preto da Universidade de São Paulo.

(4) Fonoaudióloga; Professora do Departamento de Fonoaudiologia da Universidade Federal de Santa Maria, UFSM Santa Maria, RS, Brasil; Bolsista de Produtividade 2 do CNPq; Doutora em Linguística Aplicada pela Pontifícia Universidade Católica do Rio Grande do Sul.

Conflito de interesses: inexistente de adaptação extra-uterina ${ }^{2,3}$ em virtude da imaturidade orgânica e funcional ${ }^{8,9}$, principalmente no que se refere aos sistemas respiratório, cardíaco e gastrointestinal, peso muito baixo, anemia, alterações na alimentação, além de outras dificuldades ${ }^{10}$.

Os recém-nascidos prematuros, geralmente apresentam sucção débil e anormalidades da função faríngea, que levam a dificuldades de alimentação caracterizadas por engasgo, tosse, náusea, regurgitação, refluxo faringonasal, baixo ganho ponderal, estresse respiratório e aspiração ${ }^{11}$.

Uma das causas mais frequentes das dificuldades alimentares dos recém-nascidos prematuros é a dificuldade de coordenar sucção, deglutição e respiração ${ }^{12-17}$. Distúrbios da deglutição como este podem gerar pneumonias de repetição e morte súbita ${ }^{11}$.

A assistência aos recém-nascidos tem o objetivo de favorecer o crescimento e o desenvolvimento adequados e para que isto seja possível é necessário que sejam nutridos adequadamente 2,18,19.

Para tentar contornar as dificuldades alimentares pelo uso prolongado de sonda e intubação traqueal, a realização da estimulação sensório- 
motora-oral tem sido proposta para facilitar essa transição e beneficiar a alta hospitalar 14,20,21 .

A atuação fonoaudiológica em unidades de terapia intensiva neonatal teve início na década de $80{ }^{11}$ e é feita com o objetivo de prevenir, detectar e minimizar as dificuldades relacionadas à alimentação, oferecendo melhora das condições alimentares ${ }^{22,23}$.

A estimulação da sucção não nutritiva, na qual se baseia grande parte da estimulação sensóriomotora-oral, é iniciada assim que estabilizado o quadro, principalmente respiratório e cardíaco. Para que o recém nascido pré-termo (RNPT) seja considerado estável, deve apresentar frequência cardíaca entre 120 e 160 batimentos/minuto, freqüência respiratória menor que 60 movimentos respiratórios/minuto e saturação de oxigênio maior que $90 \%$. A prioridade, após a estabilização do quadro respiratório, é o aspecto nutricional ${ }^{24}$.

Não há consenso na literatura sobre a idade adequada de se iniciar a alimentação por via oral nos prematuros ${ }^{2,16,25,26}$. Uma pesquisa utiliza como pré-requisitos estabilidade clínica, peso maior ou igual a $1500 \mathrm{~g}$ e idade gestacional igual, ou maior que 34 semanas ${ }^{27}$. Outros defendem que, geralmente, quando atingem a idade de 32 semanas tem capacidade para a sucção nutritiva, desde que esta capacidade seja precedida dos reflexos de reflexos protetores da laringe, como tosse e vômito ${ }^{11}$.

A importância da verificação dos efeitos da sucção não-nutritiva em relação aos índices de freqüência cardíaca e respiratória, assim como taxa de transferência e incremento de peso, é justificada pela escassez de estudos em relação aos parâmetros avaliados.

Além disso, uma teoria descreve a necessidade de intervenção e não estimulação de bebês com risco em virtude de estes recém-nascidos necessitarem estabilidade clínica ${ }^{25}$, avaliada nesse estudo por meio da estabilidade de freqüência cardíaca e respiratória, bem como energia suficiente para apresentar desenvolvimento motor adequado, interagir, controlar estado de consciência e aprender informações recebidas do meio, mensurada nessa pesquisa por meio da taxa de transferência e ganho de peso. ${ }^{28}$.

O controle dos índices de freqüência cardíaca e respiratória antes de depois da alimentação, são de grande importância para avaliar os benefícios da estimulação sensório motora oral em relação à estabilização destes índices, ou os possíveis prejuízos da intervenção nesta população de risco. Da mesma forma, avalia-se os benefícios da estimulação sensório-motora-oral por meio da taxa de transferência durante a alimentação, bem como ganho de peso durante internação, pela necessidade energia que esta população apresenta para desenvolver-se.

Apesar de estudos demonstrarem que a estimulação sensório-motora-oral melhora o aspecto nutricional, a maturação global, o ganho ponderal, o funcionamento do aparelho digestivo, favorecendo a taxa de transferência e propiciando a alta hospitalar precoce, a estimulação ainda não está implantada em todos os serviços de atendimento neonatal ${ }^{3}$. Dessa forma, faz-se necessário aprofundar a investigação dos benefícios que esta pode trazer aos RNPT ${ }^{11}$.

Dessa forma, o presente estudo objetiva avaliar os efeitos da estimulação sensório-motora oral de recém-nascidos pré-termo considerando índices de frequência cardíaca e respiratória pré e pós-sucção nutritiva, taxa de transferência, tempo de transição entre sonda orogástrica e via oral, além de incremento de peso durante o tempo de transição.

\section{MÉTODO}

Foi realizado um estudo transversal e de caráter quantitativo.

A coleta foi feita no banco de dados do Projeto Efeitos da Estimulação Sensório-Motora-Oral no Desempenho Nutricional de Recém-Nascidos PréTermo durante a Internação em Unidade de Tratamento Intensivo (UTI) Neonatal e sua Repercussão no Primeiro Ano de Vida, o qual tem como população recém-nascidos internados na UTI Neonatal de um Hospital Universitário.

Os critérios de inclusão e exclusão desse estudo foram os mesmos do projeto de referência.

Foram incluídos no estudo todos os recémnascidos pré-termo, entre 28 e 34 semanas de idade gestacional corrigida, determinada pelo método de New Ballard ${ }^{29}$, que se apresentassem clinicamente estáveis e cujos pais e/ou representantes legais consentissem com a inclusão no estudo e assinassem o Termo de Consentimento Livre e Esclarecido, obedecendo a Resolução MS 196/96.

Foram critérios para exclusão a presença de malformações da cabeça e pescoço, síndromes genéticas, hemorragia intracraniana grau III e IV (diagnosticadas por ultra-sonografia de crânio), asfixia perinatal (definida pela presença de Apgar de $5^{\circ}$ minuto menor ou igual a 6 ) e encefalopatia bilirrubínica diagnosticada pela equipe médica.

Além disso, foram incluídos os recém-nascidos que apresentavam protocolos de avaliação datadas entre janeiro de 2007 a junho de 2008, com preenchimento de todos os parâmetros avaliados (idade gestacional e peso ao nascimento; idade gestacional corrigida, idade cronológica e peso na primeira e segunda avaliação e volume 
prescrito e volume ingerido na primeira alimentação por via oral.

Após satisfazerem os critérios da pesquisa, os recém-nascidos foram alocados em dois grupos, mediante sorteio prévio, constituindo os grupos estimulado (GE) e controle (GC).

Os recém nascidos entravam na pesquisa ao atingir $80 \mathrm{Kcal} / \mathrm{ml} /$ dia. Durante a participação no projeto, os RNPT passaram por duas avaliações. No momento da introdução da via oral, geralmente 34 semanas, realizou-se a primeira avaliação, quando foram mensurados o peso; a idade gestacional corrigida; a idade cronológica. Nessa mesma avaliação, foram avaliados os benefícios da sucção nutritiva em relação a estabilização destes recém-nascidos, por meio dos índices de frequência cardíaca e respiratória antes e depois da sucção. Além disso, foi avaliada a taxa de transferência durante a alimentação. A segunda avaliação foi realizada após 24 horas de recebimento do alimento somente por via oral. Além dos aspectos iniciais, foram mensurados o tempo de transição entre sonda orogástrica e via oral plena e o incremento de peso durante o período.

Adotou-se como referência os valores de 120 a 160 batimentos/minuto para a frequência cardíaca e menor que 60 movimentos respiratórios/minuto para frequência respiratória ${ }^{24}$.

Além da aferição da freqüência cardíaca e freqüência respiratória referidas acima, as variáveis taxa de transferência, tempo de transição entre sonda orogástrica e via oral plena e incremento de peso baseavam-se em:

- Taxa de Transferência: volume prescrito e volume ingerido durante a primeira avaliação, realizou-se o cálculo dividindo o volume ingerido por via oral pelo volume prescrito e o resultado multiplicado por 100.

- Tempo de Transição entre Sonda Orogástrica e Via Oral Plena: foi feita com base na data da liberação da via oral (primeira avaliação) e após 24 horas de recebimento de alimento somente por via oral (segunda avaliação).

- Incremento de Peso: calculado comparando os pesos apresentados na primeira e segunda avaliação.

O grupo estimulado do projeto de referência recebeu estimulação sensório-motora-oral, duas vezes por dia a partir de seu ingresso no projeto. A estimulação sensório-motora-oral foi interrompida sempre que o recém-nascido apresentou comportamentos de retraimento como trancamento de mandíbula, aumento da frequência respiratória, cianose peri-oral, choro ou soluço.
O programa de estimulação baseou-se no proposto por Fucile et al. ${ }^{30}$ e constou de massagens extra e intra-orais feitas com a mão enluvada, seguidas da sucção não-nutritiva com o dedo enluvado, realizada por fonoaudiólogas e acadêmicas. A oferta de via oral foi feita pelas técnicas de enfermagem do serviço e os índices de frequência cardíaca e respiratória foram mensurados, antes e depois da oferta de alimento, pelas fonoaudiólogas e acadêmicas anteriormente citadas.

Este estudo está devidamente registrado no Comitê de Ética em Pesquisas (CEP) da instituição, sob o número 0131.0.243.000-06.

Os resultados obtidos foram digitados em um banco de dados e analisados por meio do software estatístico STATA (10) ${ }^{31}$. Os valores estão expressos em média e desvio padrão. Para a comparação entre os grupos utilizou-se o Teste T-Student. Foi aceito um nível de significância de $\mathrm{p}<0,05$.

\section{RESULTADOS}

Foram estudados 28 recém-nascidos prétermo, sendo 13 pertencentes ao GE e 15 ao GC. Os grupos foram semelhantes ao nascer quanto à idade gestacional e peso, bem como quanto à idade gestacional corrigida, idade cronológica e peso na primeira e segunda avaliações (Tabela 1).

Em relação ao índice de freqüência respiratória, não foi encontrada diferença estatisticamente significante entre os grupos, considerando-os antes e após a sucção nutritiva, em ambas as avaliações. Apesar disso, percebe-se que o GE manteve maior estabilidade após a alimentação na primeira avaliação em relação ao GC (Tabela 2).

Quanto à frequência cardíaca, também não foram encontradas diferenças estatisticamente significantes quando observadas antes e depois da alimentação nas duas avaliações. Porém, observase que na segunda avaliação o GE apresentou maior estabilidade do que o GC após a alimentação (Tabela 3).

Quando se refere ao volume prescrito, volume ingerido e taxa de transferência, não foram encontrados valores que diferissem estatisticamente entre os grupos, tendo estes apresentados resultados semelhantes (Tabela 4).

Em relação ao tempo de transição de alimentação por sonda orogástrica para via oral plena, o GE levou 12,62 dias para chegar a via oral plena, enquanto que o grupo controle demorou 9,47 dias. Quanto ao incremento de peso observado entre a liberação oral e o recebimento de alimento exclusivo por esta via, os grupos apresentaram aumento de peso muito semelhantes (Tabela 5). 
Tabela 1 - Caracterização dos grupos estimulado e controle ao nascimento quanto a idade gestacional e peso, e na $1^{\underline{a}}$ e $2^{\mathrm{a}}$ Avaliação Fonoaudiológica quanto a idade gestacional corrigida, idade cronológica e peso

\begin{tabular}{lccc}
\hline Variáveis & GE & GC & p \\
\hline Nascimento & & & \\
IG (semanas) & $31(+/-1)$ & $31(+/-1)$ & 0,3017 \\
Peso (gramas) & $1290(+/-295,2)$ & $1364,6(+/-341,4)$ & 0,7299 \\
1⿳亠丷厂 Avaliação & & & \\
IGC (semanas) & $35(+/-1)$ & $33(+/-7)$ & 0,1526 \\
IC (dias) & $24,1(+/-14,7)$ & $25,1(+/-10,6)$ & 0,5777 \\
Peso (gramas) & $1634,2(+/-155)$ & $1762(+/-241,6)$ & 1,6857 \\
$\mathbf{2}^{\text {a }}$ Avaliação & & & \\
IGC (semanas) & $36(+/-16)$ & $36(+/-7)$ & 0,1557 \\
IC (dias) & $35,2(+/-16,86)$ & $34(+/-10,4)$ & 0,4158 \\
Peso (gramas) & $2006,9(+/-312,8)$ & $2107,3(+/-371)$ & 0,7779 \\
\hline
\end{tabular}

T-Student, $\mathrm{p}<0,05$

GE - grupo estimulado

$\mathrm{GC}$ - grupo controle

$I G$ - idade gestacional

IGC - idade gestacional corrigida

IC - idade cronológica

Tabela 2 - Características dos grupos estimulado e controle considerando freqüência respiratória antes e depois da sucção nutritiva

\begin{tabular}{|c|c|c|c|c|c|}
\hline & \multicolumn{2}{|c|}{ GE } & \multicolumn{2}{|c|}{ GC } & \multirow[t]{2}{*}{$p$} \\
\hline & $<60 \mathrm{mr} / \mathrm{m}$ & $>60 \mathrm{mr} / \mathrm{m}$ & $<60 \mathrm{mr} / \mathrm{m}$ & $>60 \mathrm{mr} / \mathrm{m}$ & \\
\hline \multicolumn{6}{|c|}{ 1ª Avaliação } \\
\hline $\mathrm{FRA}^{*}$ & $84,62 \%$ & $15,38 \%$ & $86,67 \%$ & $13,33 \%$ & 0,877 \\
\hline $\mathrm{FRD}^{*}$ & $84,62 \%$ & $15,38 \%$ & $73,33 \%$ & $26,67 \%$ & 0,468 \\
\hline \multicolumn{6}{|c|}{ 2ª Avaliação } \\
\hline $\mathrm{FRA}^{*}$ & $100 \%$ & $0 \%$ & $100 \%$ & $0 \%$ & - \\
\hline FRD* $^{*}$ & $61,54 \%$ & $38,46 \%$ & $80 \%$ & $20 \%$ & 0,410 \\
\hline
\end{tabular}

T-Student, $p<0,05$

GE - grupo estimulado

$\mathrm{GC}$ - grupo controle

FRA - frequência respiratória antes da sucção nutritiva

FRD - frequência respiratória depois da sucção nutritiva

$\mathrm{mr} / \mathrm{m}$ - movimentos respiratórios por minuto

\section{DISCUSSÃO}

Os achados neste trabalho referem-se unicamente à estimulação sensório-motora-oral, visto que os RNPT são semelhantes nos grupos estudados. $\mathrm{Na}$ Tabela 1 observa-se que os RNPT participantes desta pesquisa apresentavam semelhanças quanto ao peso e idade gestacional ao nascimento. Quando esses RNPT foram liberados para receber a alimentação por via oral, assim como quando passaram a se alimentar apenas por via oral sem o auxílio da sonda orogástrica, mantiveram-se homogêneos quanto ao peso, à idade gestacional corrigida e à idade cronológica. Esses dados demonstram a similaridade quanto às características gerais dos RNPT ao nascimento, na liberação da via oral e na via oral plena.

$\mathrm{Na}$ pesquisa realizada por Neiva e Leone ${ }^{18}$, tanto o GE quanto os que apresentavam idade gestacional, peso ao nascimento e média idade gestacional corrigida na liberação da via oral semeIhantes aos deste estudo. Na pesquisa de Figueiredo et $\mathrm{al}^{2}$, apesar de serem semelhantes quanto à idade gestacional corrigida, os grupos tiveram diferença de peso estatisticamente significativa na 
Tabela 3 - Características dos grupos estimulado e controle considerando frequência cardíaca antes e depois da sucção nutritiva

\begin{tabular}{|c|c|c|c|c|c|c|c|}
\hline & \multicolumn{3}{|c|}{ GE } & \multicolumn{3}{|c|}{ GC } & \multirow[t]{2}{*}{$p$} \\
\hline & $<120 \mathrm{~b} / \mathrm{m}$ & $120-160 \mathrm{~b} / \mathrm{m}$ & $>160 \mathrm{~b} / \mathrm{m}$ & $<120 \mathrm{~b} / \mathrm{m}$ & $120-160 \mathrm{~b} / \mathrm{m}$ & $>160 \mathrm{~b} / \mathrm{m}$ & \\
\hline \multicolumn{8}{|c|}{ 1ªvaliação } \\
\hline $\mathrm{FCA}^{*}$ & $7,69 \%$ & $69,23 \%$ & $23,08 \%$ & $0 \%$ & $73,33 \%$ & $26,67 \%$ & 0,827 \\
\hline $\mathrm{FCD}^{*}$ & $0 \%$ & $69,23 \%$ & $30,77 \%$ & $0 \%$ & $73,33 \%$ & $26,76 \%$ & 1,0 \\
\hline \multicolumn{8}{|c|}{ 2ªvaliação } \\
\hline $\mathrm{FCA}^{*}$ & $0 \%$ & $69,23 \%$ & $30,77 \%$ & $0 \%$ & $80 \%$ & $20 \%$ & 0,670 \\
\hline FCD* & $0 \%$ & $46,15 \%$ & $53,85 \%$ & $6,67 \%$ & $40 \%$ & $53,33 \%$ & 1,0 \\
\hline
\end{tabular}

T-Student, $\mathrm{p}<0,05$

GE - grupo estimulado

GC - grupo controle

FCA - frequência cardíaca antes da sucção nutritiva

FCD - frequência cardíaca depois da sucção nutritiva

$\mathrm{b} / \mathrm{m}$ - batimento por minuto

Tabela 4 - Características da sucção nutritiva na primeira avaliação dos grupos estimulado e controle

\begin{tabular}{lccc}
\hline & GE & GC & p \\
\hline Volume Prescrito* $^{*}$ & $27,15(+/-8,86)$ & $31,55(+/-8,43)$ & 0,779 \\
Volume Ingerido* $_{\text {Taxa de Transferência (\%) }}$ & $10,07(+/-6,30)$ & $11,86(+/-8,15)$ & 0,901 \\
\hline
\end{tabular}

T-Student, $p<0,05$

GE - grupo estimulado

$\mathrm{GC}$ - grupo controle

Tabela 5 - Características dos recém-nascidos na segunda avaliação dos grupos estimulado e controle

\begin{tabular}{lccc}
\hline & GE & GC & p \\
\hline Tempo de Transição SOG-VO* & 12,62 & 9,47 & 0,096 \\
Incremento de Peso* & 358,07 & 345,33 & 0,440 \\
\hline
\end{tabular}

GE - grupo estimulado

GC - grupo controle

aquisição de via oral plena, tendo o GC maior peso que o GE.

Em relação à frequência cardíaca e respiratória apresentadas nas avaliações, não foram encontradas diferenças significantes entre o GE e GC, concordando com o estudo de Matheus, Lima e Mitre ${ }^{28}$ que consideraram o índice de saturação de oxigênio durante a sucção não-nutritiva, também não sendo encontrada diferença significativa entre os grupos.

$\mathrm{Na}$ prática clínica, o ideal seria que fossem monitoradas a frequência cardíaca e a respiratória, durante a evolução da sucção dos recémnascidos pré-termo, para que pudesse ser avaliada objetivamente a capacidade de coordenar sucção, deglutição e respiração. Além disso, pode-se adotar como critério para suspensão da oferta de via oral em determinado horário, pela instabilidade da frequência cardíaca e/ou respiratória apresentada.

Quanto à taxa de transferência apresentada na primeira avaliação, não foi encontrada diferença estatisticamente significante entre o GE e GC. O resultado encontrado foi semelhante aos da pesquisa realizada por Pickler \& Reyna ${ }^{32}$. Em outro estudo foi avaliada a eficácia da estimulação no desempenho da alimentação de RNPT, e foram encontradas taxas de transferência e de competência com significância maior no GE ao comparar com o $\mathrm{GC}^{33}$. 
No que se refere aos dias necessários para realizar a transição plena da sonda para a via oral e incremento de peso, o GE necessitou em média de 12,62 dias e teve incremento de peso de 358,07 g e o GC 9,47 dias e 345,33 g. Em outro estudo realizado na mesma unidade, não foram encontradas diferenças significantes entre os grupos quanto ao tempo de transição de sonda orogástrica para via oral e incremento de peso ${ }^{33}$.

Apesar de não terem sido considerados neste estudo, fatores limitantes patológicos, como intercorrências após o nascimento e interferências ambientais podem ter influenciado no resultado do tempo de transição plena deste estudo, uma vez que, segundo Neiva e Leone ${ }^{18}$, a velocidade da capacidade de sucção sofre a influência destas variáveis.

Com relação ao ganho de peso, estudos sugerem que a estimulação não compromete o ganho de peso dos RNPT, visto que não observaram diferenças de incremento entre os grupos, de forma semelhante ao desta pesquisa ${ }^{20,34}$. Entretanto, uma pesquisa com 18 RNPT mostrou que o grupo estimulado teve ganho de peso maior ${ }^{35}$. Para McCain ${ }^{34}$, o que poderia influenciar ainda mais a obtenção da via oral, seria o peso ao nascer.

Quando observada a taxa de transferência tem-se noção da evolução da sucção do recémnascido, bem como da capacidade de coordenar sucção e deglutição, tendo como conseqüência ganho de peso, menor tempo de transição entre sonda e via oral, além de alta hospitalar precoce.

Considera-se que resultados mais satisfatórios poderiam ser encontrados quanto à frequência respiratória e cardíaca se a observação fosse feita também durante a sucção não-nutritiva, bem como com maior número de sujeitos. Além disso, acredita-se que se a oferta da via oral fosse feita pelos profissionais participantes do projeto de referência, os resultados destas frequências, assim como taxa de transferência, poderiam ter significância estatística, já que as técnicas de enfermagem que realizavam a oferta de via oral não tinham conhecimento de tais parâmetros durante a alimentação dos RNPT.

\section{CONCLUSÃO}

Os resultados demonstram que não houve influência do programa de estimulação sensóriomotora-oral sobre as frequências cardíaca e respiratória, taxa de transferência, tempo de transição entre sonda e via oral plena e incremento de peso dos recém-nascidos estudados. Foi observada uma tendência das crianças do grupo estimulado estabilizarem a frequência respiratória após a sucção nutritiva na primeira avaliação fonoaudiológica, como também a freqüência cardíaca após a sucção nutritiva na segunda avaliação.

Sugere-se a realização de novas pesquisas relacionando a estimulação sensório-motora-oral e os índices de frequência cardíaca e respiratória, não só na sucção nutritiva, como também na nãonutritiva, a fim de evidenciar as reais condições de estimulação e recebimento da alimentação do recém-nascido.

\begin{abstract}
Purpose: to evaluate the influence of a sensory-motor-oral stimulation in preterm newborns. Method: twenty-eight preterm newborns in a neonatal intensive care unit of a University Hospital were randomly placed into stimulated and control groups. The stimulated group received a sensory-motor-oral stimulation twice a day. There were two ratings, measuring respiratory rate and heart rate, transfer tax, transition time between tube and oral feeding and weight increase. The results were analyzed through STATA 10 software, and the groups were compared through the Independent T Student Test $(p<0,05)$. Results: no statistically significant difference was found between the groups. Conclusion: there was no influence of sensory-motor-oral stimulation on the evaluated parameters.
\end{abstract}

KEYWORDS: Infant, Newborn; Premature; Infant Care; Sucking Behavior; Feeding Methods 


\section{REFERÊNCIAS}

1. Pfitscher AP, Delgado SE. A caracterização do sistema estomatognático, após a transição alimentar, em crianças prematuras de muito baixo peso. Rev. Soc. Bras. Fonoaudiol. 2006; 1(4):215-22.

2. Figueiredo H, Barreiros J, Gonçalves I, Cunha M. Avaliação de um Programa de Intervenção Oral em Recém-nascidos Pré-termo de Muito Baixo Peso. EssFisiOnline, 2007 vol. 3, oㅡ 2 p. 3-12. disponível em: http://www.ifisionline.ips.pt/Arquivos_EssFisio_ files/vol3n2.pdf

3. Costa CN, Lima GRS, Jorge RM, Malta RACG, Nemr K. Efetividade da Intervenção Fonoaudiológica no Tempo de Alta Hospitalar do Recém-Nascido Pré-Termo. Rev CEFAC. 2007; 9(1): 72-8.

4. Castro AG, Lima MC, Aquino RR, Eickmann SH. Sensory oral motor and global motor development of preterm infants (original title: Desenvolvimento do sistema sensório motor oral e motor global em lactentes pré-termo). Pró-Fono Revista de Atualização Científica. 2007; 19(1): 29-38.

5. Fujinaga $\mathrm{Cl}$, Scochi CGS, Santos CB, Zamberlan NE, Leite AM. Validação do conteúdo de um instrumento para avaliação da prontidão do prematuro para início da alimentação oral. Rev. Bras. Saúde Matern. Infant. 2008; 8(4):391-9.

6. Bauer, Prade LS, Keske-Soares M, Haëffner LSB, Weinmann ARM. The oral motor capacity and feeding performance of preterm newborns at the time of transition to oral feeding. Braz $\mathrm{J}$ Med Biol Res. 2008; 41(10): 904-7.

7. Alves AML, da Silva EHA, de Oliveira AC. Desmame precoce em prematuros participantes do Método Mãe Canguru. Rev Soc Bras Fonoaudiol. 2007; 12(1):23-8.

8. Neiva FCB, Leone CR. Sucking in preterm newborns and the sucking stimulation (original title: Sucção em recém-nascidos pré-termo e estimulação da sucção). Pró-Fono Revista de Atualização Científica. 2006; 18(2):141-50.

9. Santoro Júnior W, Martinez FE. Effect of intervention on the rates of breastfeeding of very low birth weight newborns. J Pediatr. 2007; 83(6):541-6. 10. Scotegagna AS, Miranda CA, Morsch DS, Carvalho RA, Biasi J, Cherubini F. O processo interativo mãe bebê pré-termo. Rev. Psic. Vetor Ed. 2005; 6(2):61-70.

11. Rios IJA. Estimulação de sucção para recém-nascido de alto risco. In: Marchesan, IQ. Fundamentos em fonoaudiologia. 2. ed. São Paulo: Guanabara Koogan; 2005. p. 11-7.

12. Aquino RR, Osório MM. Alimentação do recém-nascido pré-termo: métodos alternativos de transição da gavagem para o peito materno. Rev. Bras. Saúde Matern. Infant. 2008;8 (1): 11-6.

13. Amaizu N, Shulman RJ, Schanler RJ, Lau C. Maturation of oral feeding skills in preterm infants. Acta Paediatr. 2008; 97(1): 61-7.

14. Bauer MA, Yamamoto RCC, Keske-Soares M. Evaluation of sensory-motor-oral in the transition from gastric tube to full oral feeding in preterm newborn. Ver. Bras. Saúde Matern. Infant. 2009; 9(4):429-34.

15. Yamamoto RCC, Keske-Soares M, Weinmann ARM. Características da sucção nutritiva na liberação da via oral em recém-nascidos pré-termo de diferentes idades gestacionais. Rev Soc Bras Fonoaudiol. 2009;14(1):98-105.

16. Yamamoto RCC, Bauer MA, Häeffner LSB, Weinmann ARM, Keske-Soares M. Os efeitos da estimulação sensório motora oral na sucção nutritiva na mamadeira de recém-nascidos pré-termo. Rev. CEFAC. 2009. disponível em: http://www.scielo.br/ pdf/rcefac/2009nahead/117-08.pdf.

17. Rossarolla C, Menon MU, Scochi CGS, Fujinaga $\mathrm{Cl}$. Validade discriminatória do instrumento de avaliação da prontidão para início da alimentação oral de bebês prematuros. Rev Soc Bras Fonoaudiol. 2009;14(1):106-14.

18. Neiva FCB, Leone CR. Sucção em recémnascidos pré-termo e estimulação da sucção. Pró-Fono. 2006; 18(2):141-50.

19. Hernandez AM, Giordan CR, Shiguematsu RA. A intervenção fonoaudiológica em recém-nascidos de risco para distúrbios da deglutição e sua influência no aleitamento materno. Rev Bras Nutr Clin 2007;22(1):41-4.

20. Rocha AD, Moreira MEL, Pimenta HP, Ramos JRM, Lucena SL. A randomized study of the efficacy of sensory-motor-oral stimulation and non-nutritive sucking in very low birthweight infant. Early Hum Dev. 2007; 83(6):385-8.

21. Boiron M, Roux S, Henrot A, Saliba E. Effects of oral stimulation and oral support on nonnutritive sucking and feeding performance in preterm infants. Dev Med Child Neurol. 2007; 49:439-44.

22. Marilene de Souza Rocha1, Susana Elena Delgado2. Intervenção fonoaudiológica em recémnascido pré-termo com gastrosquise. Rev Soc Bras Fonoaudiol. 2007;12(1):55-62.

23. Neiva FCB, Leone CR. Development of sucking rhythm and the influence of stimulation in premature infants (original title: Evolução do ritmo de sucção e influência da estimulação em prematuros). Pró-Fono Revista de Atualização Científica. 2007; 19(3): 241-8.

24. Xavier $C$. Intervenção fonoaudiológica em bebês de risco. In: Ferreira, LP; Befi-Lopes D, Limongi S. 
Tratado de fonoaudiologia. São Paulo: Roca; 2004. p. 415-38.

25. Neiva FCB, Leone CR. Efeitos da estimulação da sucção não-nutritiva na idade de início da alimentação via oral em recém-nascidos pré-termo. Rev Paul Pediatria 2007;25(2):129-34.

26. Fujinaga $\mathrm{Cl}$, Zamberlan NE, Rodarte MDO, Scochi CGS. Reliability of an instrument to assess the readiness of preterm infants for oral feeding (original title: Confiabilidade do instrumento de avaliação da prontidão do prematuro para alimentação oral). Pró-Fono Revista de Atualização Científica. 2007; 19(2): 143-50.

27. do Nascimento MBR, Issler $\mathrm{H}$. Aleitamento materno em prematuros: manejo clínico hospitalar. J Pediatr. 2004; 80(5):163-72.

28. Matheus J, Lima M, Mitre E. Efeitos da sucção não nutritiva em recém nascidos pré-termo considerando índice de saturação de oxigênio. Rev. CEFAC. 2004; 6(3):282-7.

29. Ballard JL, Khoury JC, Weding K, Wang L, Eilers-Waisman BL, Lipp R. New Ballard Score, expanded to include extremely premature infants. $J$ Pediatr. 1991; 119(3):417-23.

30. Fucile S, Gisel E, Lau C. Oral stimulation accelerate the transition from tube to oral feeding in preterm infants. J Pediatr. 2002;141:230-6.

31. Stata Versão 10. Stata press publication. College Station Texas: Stata Corpe LP; 2007.

32. Pickler RH, Reyna BA. Effects of non-nutritive sucking on nutritive sucking, breathing, and behavior during bottle feeding of preterm infants. Adv Neonatal Care. 2004; 4(4):226-34.

33. Höher FP. Estimulação sensório-motora-oral e o desempenho nutricional de recém-nascidos pré-termo. [dissertação] Santa Maria (RS): Universidade Federal de Santa Maria; 2005.

34. McCain GC. An evidence-based guideline for introducing oral feeding to healthy preterm infants. Neonatal Network. 2003; 22:45-50.

35. Gaebler CP, Hanzlik JR. The effects of a prefeeding stimulation program on preterm infants. Am J Occup Ther. 1996; 50(3):184-92. 